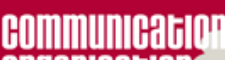

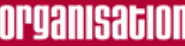

\section{Communication et organisation}

Revue scientifique francophone en Communication organisationnelle

39 | 2011

Les applications de la sémiotique à la communication des organisations

\title{
Approche socio-sémiotique de la relation client
}

\section{Christiane Legris-Desportes}

\section{(2) OpenEdition}

\section{Journals}

Édition électronique

URL : https://journals.openedition.org/communicationorganisation/3102

DOI : 10.4000/communicationorganisation.3102

ISSN : $1775-3546$

\section{Éditeur}

Presses universitaires de Bordeaux

\section{Édition imprimée}

Date de publication : 1 juin 2011

Pagination : 125-136

ISBN : 978-2-86781-744-1

ISSN : $1168-5549$

\section{Référence électronique}

Christiane Legris-Desportes, " Approche socio-sémiotique de la relation client », Communication et organisation [En ligne], 39 | 2011, mis en ligne le 01 juin 2014, consulté le 28 juin 2022. URL : http:// journals.openedition.org/communicationorganisation/3102; DOI : https://doi.org/10.4000/ communicationorganisation.3102 


\title{
Approche socio-sémiotique de la relation client
}

\author{
Christione Legris-Desportes ${ }^{1}$
}

Sous l'impact conjugué des innovations technologiques et de l'évolution des tendances et pratiques socio-culturelles, les phénomènes de prise de parole des organisations connaissent des transformations et de nouvelles formes relationnelles se développent entre entreprises, clients (ou prospects) et individus. La « relation client », processus à la fois technique, organisationnel et communicationnel, composante stratégique majeure dans les organisations développant des offres de services et de produits à destination de différents publics, met en ouvre des modalités communicationnelles qui s'inscrivent dans des formes sémiotiques - de plus en plus ? - complexes, et dont seule une approche pluridisciplinaire permet de rendre compte. La question majeure qui sous-tend cet article porte ainsi sur la construction et la mise en perspective d'une socio-sémiotique de la relation au client à des fins compréhensives. Socio-sémiotique est entendue ici comme l'étude de la production de signes dans le champ des interactions sociales (Landowski, 1989) et, conséquemment, la prise en compte des conditions de manifestation des discours avec un élargissement à l'analyse des problématiques de type socio-culturel. L'approche ne se limite donc pas au démontage des mécanismes textuels et à la description de leur niveau, à énumérer valeurs et oppositions internes des discours, mais prend en compte :

«...des problématiques plus générales, qui concernent les raisons de l'apparition d'un certain discours, sa position dans le cadre de la discursivité sociale et des mécanismes de circulation et de réception qui permettent de comprendre la trajectoire de ce discours, son éventuel succès ou insuccès, sa prise en charge ou sa marginalisation de la part des acteurs sociaux ${ }^{2}{ }^{2}$.

L'analyse des textualités - de leurs modalités discursives et formelles produites dans le contexte de la relation au client s'envisage alors en intégrant une réflexion sur la contemporanéité dans laquelle elles se construisent et se

1. EDF - Recherche\&Développement. Chercheure au CERES - Centre de Recherche Sémiotique- Université de Limoges. christiane.legris-desportes@edf.fr

2. Andrea Semprini, Analyser la communication 2, Paris, L'Harmattan, coll. "Champs Visuels », 2007, p.16,17. 
déploient. Ainsi, les pratiques communicationnelles des entreprises en matière de relation client renvoient aujourd'hui à diverses thématiques que nous nous proposons d'investiguer, et notamment celles de manifestation du lien social et de rapport à la transparence. Mais avant d'appréhender ces différentes notions - qui, on va le voir, ouvrent elles-mêmes sur des problématiques spécifiques et de nature pluridisciplinaire - une question centrale se pose, en amont : celle des hétérogénéités médiatiques et discursives, qui trouvent une actualisation accrue en contexte de médiation numérique de la communication organisationnelle.

Nous développerons ces points en commençant par situer les apports de la socio-sémiotique à l'analyse des pratiques discursives et organisationnelles de la relation client, tout en cherchant à montrer en quoi et comment les analyses évoluent nécessairement, tant en termes de problématiques que sur le plan méthodologique.

\section{Les problématiques socio-sémiotiques qui se posent en entreprise}

On sait depuis longtemps qu'il existe " une rhétorique dont le lieu spécifique est le marché » (Bordron, 1999) et que les entreprises n'organisent pas seulement la production de biens matériels mais qu'elles assurent également la gestion du sens qu'elles produisent consciemment ou inconsciemment (Fraenkel et Legris-Desportes, 1999). Cet actif immatériel s'est considérablement intensifié avec, ces dernières années, une prééminence, dans les communications commerciales, des «imaginaires de marque, des émotions communes, des relations humaines, des interactions symboliques et des styles de vie » (Huygues, 2004). Les bouleversements technologiques des dernières décennies et l'évolution de certaines tendances socio-culturelles ont profondément modifié les pratiques relationnelles entre les différents acteurs de la relation marchande. Du côté des entreprises, la généralisation du service en ligne a conduit à partir des années 1990 à l'apparition de nouveaux métiers et de nouvelles instances (les conseillers en ligne et les centres d'appel, les webmasters,...) et bien sûr, les formes d'interactivité et de discursivité se sont renouvelées : création d'argumentaires commerciaux, de pages internet, ouverture et alimentation de forums, etc. Aujourd'hui, de nouveaux outils virtuels sont régulièrement proposés, comme par exemple l'introduction récente dans des entreprises françaises des jeux en ligne, venant enrichir - et complexifier-les relations que l'entreprise construit à destination de ses clients ou prospects mais également en interne. Des advergames (jeux véhiculant des messages publicitaires) sont conçus pour renforcer le capital de notoriété des émetteurs en s’inscrivant dans une stratégie de marque : produits dans l'objectif de promouvoir un produit, de fidéliser une clientèle, ils peuvent aussi s'insérer dans une démarche d'engagement sociétal, en témoignant des valeurs et engagements de l'entreprise dans telle ou telle cause. Les serious games, jeux à portée pédagogique, sont élaborés pour devenir des outils de formation 
utilisés pour accompagner la montée en compétence des employés tant dans le domaine des savoir-faire que dans celui des savoir-être. L'introduction de ces jeux, dans lesquels la notion de score individuel, de réussite, peut être présente en permanence, crée ainsi une rupture par rapport aux modalités traditionnelles d'évaluation immédiate des processus de formations, où, sur le plan institutionnel, seule la présence, la participation, était évaluée et non la performance. Par ailleurs, le développement des «médias sociaux » (les blogs, Facebook, Twitter, ...) génère lui aussi de la complexification, l'avènement du web participatif contribuant à un accroissement des parties prenantes et à de nouvelles capacités de mobilisation chez les individus (Reguer, 2009) : augmentation de leur pouvoir de prescription, de contestation et de dénonciation, dans un contexte déjà ancré de connaissances des consommateurs du fait des portails de recherche personnalisée et des flux RSS qui permettent aux internautes, depuis de nombreuses années déjà, l'accès à une information actualisée en permanence (Fichy, 2008).

Le champ de la relation clientèle s'est ainsi enrichi de nouvelles problématiques ou de nouvelles actualisations de ces dernières, avec, tout particulièrement, la question des effets de l'hétérogénéité médiatique (le multi-canal et la prolifération des contenus, l'hétéroglossie et l'imaginaire linguistique ${ }^{3}$, la cohérence de la figure d'émetteur). Les évolutions des formes de tangibilité des échanges (en lien avec ce qui est communément nommé " dématérialisation de la relation ») interrogent la nature même des interactions. En effet, le phénomène du multi-canal - et les évolutions qu'il implique pour les supports de communication - génère une multiplicité des prises de parole et une évolution de la dynamique des échanges, la communication d'entreprise continuant de se décliner sous des formes classiques (le mailing papier, le point de vente, la publicité télévisuelle, l'affichage) et à travers des supports plus récents (l'e-mailing, le concept store, le marketing mobile, les nouvelles formes de communication dans la ville : street marketing, affichage interactif, salon et magasin éphémères, etc.). Sous-tendu par une volonté de réduction des coûts (diminution des contacts en face à face, automatisation des procédures, etc.), l'accès à une version dite dématérialisée des relations commerciales a été généralisé, avec l'instauration de propositions d'e-procédures. La facture électronique remplace l'envoi papier, l'échange de mails se substitue au dialogue en face à face ; l'écriture ou la lecture d'un post sur un blog ou sur un forum ainsi que les réponses publiées par les internautes ou les membres de l'entreprise, tissent de nouvelles relations, basées sur des rencontres virtuelles et non physiques. Mais peut-on pour autant dire que ces pratiques s'inscrivent dans l'immatérialité ? Philippe

3. «Imaginaire linguistique » entendu ici en référence à sa théorisation par A.M. Houdebine. 
Quinton apporte une réponse : "écrire est quelque chose qui se voit " ${ }^{4}$, les espaces médiatiques électroniques s'inscrivent dans une matérialité technologique et iconique incontestable : une mise en visibilité de contenus linguistiques et iconiques par la médiation de l'écran. Ils ne dématérialisent pas la relation, mais viennent plus exactement lui substituer d'autres formes de tangibilité. Loin d'être immatériels, ces espaces donnent lieu à des expressions et des perceptions à la fois visuelles et phoniques (si l'émetteur incorpore des vidéos). La combinaison de ces différents supports conduit à placer la relation à la fois sur le plan de la réactivité (répondre de façon immédiate à un besoin ou une attente) et sous le signe de la permanence (accès possible dès la connexion), de la durée. Les évolutions technologiques de la relation client ont ainsi conduit à des évolutions de la temporalité dans laquelle se situent les contacts (durée versus instantané), ces derniers devant être réalisés en fonction de situations-clefs du client, et pouvant s'exercer de façon systématique ou pas, au regard des types de services auxquels ils vont permettre d'accéder. En terme d'innovation sémiotique (émergence de nouvelles formes signifiantes, Legris-Desportes, Tsala, 2008), la relation client, en tant que manifestation de contact entre deux entités distinctes - l'entreprise et ses acteurs internes versus un ou des individus externes - s'est vue investie de propriétés (et de valeurs) de mouvance, d'immédiateté et de permanence, qui ont modifié les fondements même de la dynamique interactionnelle, ce qui amène Laure Célérier à se demander ce que devient la relation, « quand les liens peuvent outrepasser la présence physique de l'autre ", et à parler de " mise en place d'un nouvel ordre sémiotique, pour repenser les concepts de lien social et de relation ». Un support de communication, un mode de contact ne sont jamais appréhendés, jugés, strictement au regard de leurs fonctionnalités, mais bien par rapport à la façon dont la relation va se réaliser sur le plan de l'interaction, la personnalisation et la proximité qu'ils vont engendrer dans la réalisation d'un acte (accéder à une information, obtenir une réponse, etc.). Ce ne sont pas seulement des médias pluriels, mais bien un dispositif relationnel singulier, aux dimensions polysensorielles (langagières, iconiques, auditives), conjointement interactives, connotatives et qui s'appréhendent de façon systémique (en tant que système). L'hétérogénéité médiatique (au sens sémiotique du terme), si elle est bien gérée, peut devenir un mode de structuration du positionnement de l'entreprise. On peut ainsi souligner l'intérêt d'une approche complémentaire en sciences de l'information et de la communication qui prenne en compte les fondements perceptifs qui organisent les représentations et les perceptions du client, à travers notamment des enquêtes qualitatives : appréhender les critères d'appréciation et les différents "objets référentiels" (tout ce qui est à la périphérie des contacts client/entreprise) qui vont amener le client à se

4. Philippe Quinton, "Ecriture, Spécificités morphologiques des écrits électroniques », Actes du colloque Ecriture en ligne : Pratiques et communautés, Sous la direction de Brigitte Chapelain, Université de Rennes 2. CERCOR (CERSIC), Septembre 2002, p. 23. 
forger une opinion, un ressenti, et à avoir bien sûr ainsi des attentes ou des déceptions en matière de modes de contact et manifestation de la relation. Nous pensons plus particulièrement aux perceptions, représentations et à l'imaginaire associés à chaque mode de contact ou type de support, aux logiques d'usage et aux pratiques en matière de services, ainsi, enfin, qu'aux critères fondamentaux d'appréciation de la qualité des services (le « système de valorisation » à travers lequel le client appréhende un service et juge de sa qualité). Cette identification des attributs qui font dire aux clients d'un service qu'il est de qualité est essentielle car par transposition, ces données permettront d'appréhender si la gestion médiatique de la relation favorise concrètement, de par ses caractéristiques, la perception d'une qualité : si le support, le média, possède symboliquement les attributs qui feront dire d'un service qu'il est de qualité, ou s'ils permettent concrètement, de par leurs caractéristiques formelles, de favoriser la qualité d'un service (nous pensons notamment à la permanence d'accès, l'immédiateté et la personnalisation). Une entreprise positionnée sur de la proximité relationnelle émet un discours contradictoire si les modes de contacts proposés à sa clientèle s'avèrent favoriser la virtualité des échanges aux dépens d'une proximité telle que celle permise dans les points d'accueil physique. Ces approches viennent en complément de celles qui consistent à analyser le canal en partant d'une requête-client : suivre étape par étape comment un client, confronté à un besoin, appréhende et utilise les différents modes de contacts qui s'offrent à lui.

\section{L'exploration sémiotique de l'hétérogénéité discursive}

Pour le sémioticien qui travaille sur la communication d'entreprise et ce que l'on nomme en marketing la gestion de la relation client, la question de l'hétérogénéité discursive se pose d'emblée, ainsi que celle, corollaire, de la polyphonie, du fait del'existence de tout un ensemble de productions langagières émises par des émetteurs multiples. Il suffit pour éclairer notre constat de citer quelques types de documents pouvant intervenir dans la constitution d'un corpus : les écrits de la relation commerciale (courriers, brochures, ...), les discours professionnels in situ (entretiens entre conseillers en ligne et différents segments de clientèles : clients particuliers et professionnels) mais également toutes les formes de discours dits de marque (la communication publicitaire diffusée par la biais de plusieurs canaux : la radio, la télévision, etc.). Par ailleurs, on vient de l'évoquer, ces formes traditionnelles de communication côtoient depuis une quinzaine d'années maintenant d'autres formes de prises de parole institutionnelles nées de l'essor des nouvelles technologies numériques : les mailings, les sites internet, les forums et les blogs, les SMS. Dans un contexte de plus en plus concurrentiel et d'internationalisation des marchés, la gestion de la relation client - ou GRC - a peu à peu constitué pour les entreprises un véritable enjeu économique, devenant parfois aujourd'hui un des seuls éléments différenciants dans des secteurs où l'offre reste, elle, peu différenciée 
(car peu différenciable). Beaucoup d'entreprises ont progressivement mis en place des outils qui rationalisent la relation client (bases de données de contacts renseignées en continu, mutualisation de la connaissance client par le reporting des actions commerciales, etc.). Mais au-delà des approches techniques et organisationnelles (relatives au développement des outils, du multi-canal, des systèmes d'informations, etc.), la qualité de la relation client passe par un dispositif technologique et relationnel qui nécessite de concilier deux niveaux : d'une part celui de la cohérence et de la complémentarité des différents supports et productions langagières et iconographiques émises par l'entreprise, et d'autre part celui du positionnement relationnel et de sa circonscription en terme de valeurs et d'imaginaires (gestion de la dimension symbolique). La production des différents textes et discours professionnels nécessite des connaissances précises sur les différents domaines du secteur d'activité de l'entreprise et de sa commercialisation (communication commerciale), mais également des stratégies d'argumentation appropriées et des postures énonciatives pertinentes : positionnement stable de l'émetteur, du récepteur et des objets référentiels, complémentarité des discours au sens de spécificité (et non seulement redondance) associée à une adéquation à la posture de marque. La socio-sémiotique contribue à alimenter la réflexion de par les méthodologies mises en oeuvre dans ses approches et les questionnements auxquels elle permet de répondre. Qu'il s'agisse pour une entreprise de développer le multi-canal ou encore de faire évoluer sa stratégie relationnelle, elle apporte en effet des grilles d'analyse qualitative qui enrichissent la réflexion et aident les praticiens en entreprise. En termes de dysfonctionnement du positionnement relationnel, une même entreprise émettrice peut construire des figures de récepteurs antagonistes, ou plus ou moins valorisantes, selon les supports. Ainsi, par exemple, tant au plan du contenu que de l'expression, certaines postures énonciatives construisent dans les documents commerciaux, et tout particulièrement dans les mailings, une figure de client en tant que sujet exécutant, dont les actions ne sont que le fait de programmations du service émetteur (telle ou telle entité de l'entreprise) : un important recours à l'impératif, un ton incitatif (un devoir faire), une redondance calculée de tous les codes de la pression commerciale, et en ce qui concerne la figure de l'entreprise, une image d'hégémonie, basée sur l'uni-directionalité de la relation. Or d'autres discours, émanant d'une même entreprise mais d'un autre support, peuvent inversement mettre en scène un récepteur actif, engagé dans une relation d'échanges et de convivialité, et construire une image d'entreprise auxiliaire au sens sémiotique du terme. Un dispositif d'expertise instrumentalisant l'analyse sémio-linguistique à des visées de diagnostic permet de prendre en compte ces questions.

Ce type de dispositif s'applique tout particulièrement à l'étude de la communication entre conseillers en ligne et clients : analyse de conversations à des visées d'optimisation des argumentaires produits en interne et plus 


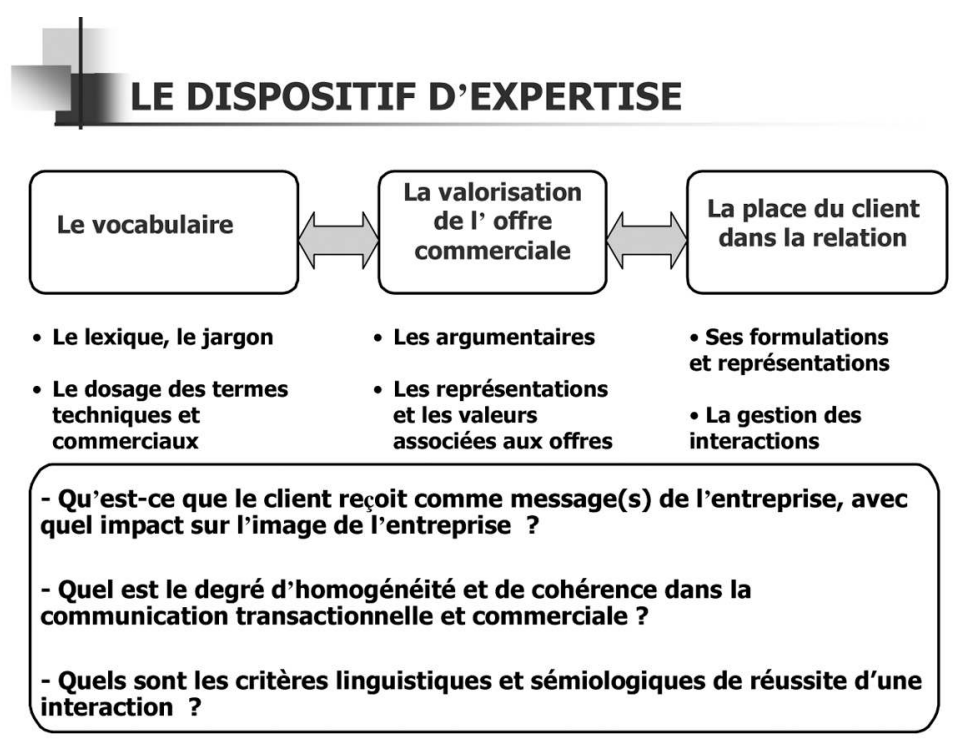

généralement, de compréhension des facteurs favorisant la réussite de la relation, la satisfaction du client. Il repose sur trois entrées centrales du fait des problématiques sur lesquelles elles ouvrent : la question du vocabulaire et plus généralement du sociolecte comme source potentielle d'incompréhension des clients ; les modalités de valorisation des offres de services ou de produits et, par exemple, l'adéquation des bénéfices mis en avant au positionnement de marque ; enfin, la place laissée au client dans la relation, avec deux niveaux d'interrogation : les temps d'expression donnés au client - ou pas - dans l'interaction et la prise en compte par le conseiller des formulations et façons d'appréhender propres au client pour construire ses propres réponses. La pratique d'une socio-sémiotique appliquée à des corpus d'entreprise donne ainsi lieu à la production de différents dispositifs d'analyse, élaborés pour appréhender les aspects communicationnels et organisationnels en se basant sur les problématiques telles qu'elles se posent en entreprise.

\section{Les social communities et leurs conséquences interactionnelles dans la relation client : une valorisation accrue de la transparence}

Les réseaux sociaux sur lesquels les internautes, clients d'une entreprise, viennent spontanéments'exprimer jouent un rôle de plus en plus important dans la construction des savoirs collectifs et individuels sur une marque, un produit, un service, et à ce titre, ils impactent directement la relation qui s'instaure entre les entreprises et les clients (ou prospects). Les blogs et les forums, à l'instar des sites Internet, sont des lieux de consignation d'informations mais aussi et surtout des espaces de confrontation entre des discours de l'entreprise et des expériences concrètes des individus. En terme de communication, 
ce sont des lieux où se dressent des constats, positifs ou négatifs, où se valident ou s'invalident des promesses d'entreprises, où se développent des contre-argumentations, tant en matière de services et produits qu'en matière d'éthique et de responsabilité sociale. Ces espaces de discours viennent influencer les processus de décision des consommateurs et les entreprises doivent élaborer des communications qui intègrent une stratégie de réponse, à la fois commerciale et sociale (nous reviendrons ci-après sur cette question de réponse sociale et d'engagement sociétal). Le développement de ces communautés s'inscrit certes totalement dans des rationalités déjà analysées en sciences de l'information et de la communication, l'homogamie restant un critère déterminant dans les relations que les internautes tissent entre eux (forums sur la santé, sur telle ou telle question sociétale spécifique,...), mais leurs pratiques engendrent de nouveaux enjeux communicationnels dans les organisations en matière de stratégie de dialogue, avec des interrogations sur la régulation versus l'auto-régulation des prises de paroles des internautes.

La prolifération des " technologies sociales " telles que Facebook et Twitter a également entraîné une évolution sans précédent de la dimension interactionnelle de la relation client, un effet de contagion donnant notamment naissance à des plates-formes collaboratives. Le développement des «social communities ", en offrant aux clients des espaces d'expression pour poser des questions, proposer des solutions originales, etc. vient bouleverser les formes interactionnelles traditionnelles et introduire une notion de relation dialogique et de co-construction de l'offre.

En parallèle, les études qualitatives menées auprès des consommateurs et le suivi des blogs socio-culturels montrent que les attentes des formes d'engagement sociétal des entreprises (notamment à travers le concept de RSE, Responsabilité Sociale des Entreprises) évoluent, avec des attentes de transparence très forte de la part des individus. En effet, en ces temps de crises (crise sociale, crise financière, et maintenant crise nucléaire...) qui réactualisent les imaginaires négatifs d'insécurité, de remise en cause, de secret voire de conspiration, avec des connotations très anxiogènes, la transparence constitue de plus en plus une valeur de prédilection. La crise financière était déjà venue conférer un «nouveau » sens à la consommation, le consumérisme des décennies antérieures et les valeurs d'abondance ayant laissé place à de nouvelles représentations (Legris, 2010), celles d'une consommation plus responsable sur le plan individuel et collectif5. L'empowerment (ou réappropriation d'un pouvoir personnel par les individus, une capacité d'action), envisagé par certains comme à la base d'un nouveau contrat social depuis quelques années déjà ${ }^{6}$, semble s'étendre, en tous les cas dans une

5. Même si bien sûr il s'agit là d'une évolution des modes de consommation amorcée depuis plus d'une dizaine années.

6. Cf par exemple article de la Chaire Unesco, publié en ligne le 12/09/08, http://chaire-urbademo.com/ dossiers/dossiers.phb 
acception socio-psychologique du terme, donnant lieu à un renouvellement des formes d'action et d'engagements à la fois individuels et collectifs. Inévitablement, ces évolutions impactent le positionnement et les prises de parole des entreprises, qui au-delà d'une promesse émotionnelle, doivent actualiser leur engagement sociétal à travers un discours sur leurs actions et les valeurs qui les mobilisent.

\section{Exploration de la dimension socio-culturelle}

Au-delà d'une " connaissance client » correspondant à la somme des informations qu'une entreprise peut récolter au fur et à mesure des contacts qui s'instaurent (alimentation de base de données, programme de fidélisation, etc.), les grandes entreprises cherchent souvent à disposer d'informations sociologiques (qualitatives et quantitatives) sur leur clientèle, celles-ci généralement ordonnées autour de trois grands types de données :

- L'univers des services clients, les perceptions et les attentes de la clientèle sur les services et les offres offerts par l'entreprise ou par des concurrents (les composantes de la satisfaction et de la fidélisation).

- La perception de l'entreprise, de ses messages, de l'image qu'elle véhicule (l'impact de la communication corporate, mais plus généralement les images perçues du positionnement de marque).

- Le contexte socioculturel : la connaissance des pratiques et des tendances, des évolutions sociétales et des valeurs dans l'air du temps, cette connaissance dépassant totalement le cadre des clients de l'entreprise pour s'appliquer à la notion de consommateurs, d'individus dans une société donnée (les modes de consommation par exemple, mais aussi les usages, etc.).

Les études centrées sur le contexte socioculturel s'attachent à comprendre quelles sont les tendances, les valeurs dans l'air du temps (émergentes mais également ancrées) qui viennent orienter les comportements. Il s'agit, à partir d'analyses sociologiques et sémiologiques, de mettre en place des veilles tendancielles. Les études sémiologiques portent le plus souvent sur les discours médiatiques, publicitaires et spécialisés, et prennent également comme objet d'analyse les " produits socio-culturels » tels que les films, les romans, etc. Il est évident que cette composante d'analyse est fondamentale car elle permet aux entreprises d'appréhender et de mesurer les impacts du contexte socioculturel sur les attentes et les comportements, et par là-même, de développer des offres et des moyens de portage plus cohérents (en connaissant mieux la dimension sociétale). Ainsi, par exemple, les études de tendances ayant montré il y a quelques années déjà l'émergence d'un fort besoin - et d'une capacité - d'expression personnelle (il n'est qu'à constater aujourd'hui l'essor des blogs...) ainsi qu'un besoin de maîtrise des choix (se faire une vie qui a du sens pour soi), les entreprises ont, dans leur gestion de la relation à la clientèle et dans leurs offres, développé personnalisation et sur-mesure, le fameux « one to one ». Elles ont également développé des 
services de facilitation de la vie quotidienne, facilitation d'accès physique et mental. Dès les années 2000, on a commencé à observer le passage progressif d'un système de référence dominant : posséder et accumuler des biens, la réussite sociale, à un autre : une relativisation de la place du travail et de l'argent, une nouvelle place de l'éthique, etc. Il est primordial de tenir compte de cette mutation des valeurs en cherchant à la traduire en services et en « composantes », y compris dans les modalités relationnelles de l'entreprise avec sa clientèle. Ainsi, ces derniers doivent tout particulièrement ne pas s'apparenter à du harcèlement commercial, et satisfaire au besoin croissant de relationnel dans le transactionnel. Le repérage des tendances ne permet pas seulement de proposer des produits ou des services innovants, d'adopter une vision dynamique du marché en saisissant le sens de l'évolution des comportements et des valeurs; il aide également à mieux spécifier la relation clientèle en l'adaptant aux attentes des publics.

\section{Conclusion}

Les pratiques communicationnelles et organisationnelles qui entrent dans le cadre des relations stratégiques que les entreprises entretiennent et développent avec leur clientèle ont donné naissance dans le passé à une notion spécifique, celle de « relation client ». Celle-ci, peu à peu investie par d'autres disciplines que le marketing, constitue aujourd'hui un objet d'étude à part entière chez certains socio-sémioticiens. La pratique d'une socio-sémiotique appliquée à des corpus d'entreprise donne ainsi lieu à la constitution de différents dispositifs d'analyse, élaborés pour appréhender les aspects communicationnels et organisationnels en se basant sur les problématiques telles qu'elles se posent en entreprise. Parmi celles-ci, la question de la gestion de l'hétérogénéité et de la cohérence est centrale. Les formes d'interactivité et de discursivité se sont inévitablement renouvelées dans les organisations, accompagnant les évolutions techniques, le développement d'usages autour des réseaux sociaux et un certain renouvellement de tendances socio-culturelles. De ce fait, les pratiques relationnelles entre les différents acteurs de la relation marchande se sont complexifiées. La multiplicité des supports de communication, la création d'outils virtuels, conjuguées à des attentes spécifiques de la part des clients (ainsi notamment d'un relationnel inscrit dans plus de transparence, d'un positionnement de marque témoignant d'un projet sociétal plus éthique en lien avec la notion de RSE) rendent le regard socio-sémiotique de plus en plus légitime en entreprise. 


\section{BIBLIOGRAPHIE}

BERTRAND D. et FONTANILLE J., Figures et régimes sémiotiques de la temporalité, PUF, 2006.

BITOUN C. et LEGRIS-DESPORTES C., «Analyse de sites Internet », in Analyser la communication 2 (sous la direction d'Andrea Semprini), L'Harmattan, 2007.

BOUTAUD J.-J., Transparence, Communication, L'Harmattan, 2007.

BRUGIDOU M. et al., "Les facteurs de choix et d'utilisation de logiciels d'Analyse de Données Textuelles ", actes de colloque $5^{e}$ Journées Internationales d'Analyse Statistique des Données Textuelles, JADT, 2000.

BROWN S., Torment Your customers, they'll love it, Harward Business Review, october, 2001.

COVA B., LOUYOT M.C. et LOUIS-LOUISY M., Les innovations marketing en réponse à la montée de l'hédonisme: Articulations avec le CRM ?, $3^{\text {ème }}$ Congès sur les tendances du marketing en Europe, 2003.

CÉLÉRIER L., « Signe, culture et lien social à l'ère des réseaux », Degrés, n 126-127, 2007 », Lectures [En ligne], URL : http://lectures.revues.org/470, 2007.

DESJEUX D., La consommation, PUF et http://www.argonautes.fr/sections. php?op=, 2008.

DUONG Q.L., " La RSE des entreprises, pourquoi et comment ça parle », Communication et Organisation 26, 2004.

FLOCH J.-M., Sémiotique, marketing et communication, PUF, 1990.

GAZIER B., Le modèle social à l'épreuve de la crise, Rencontre La Prospective, 2009.

HAMBURSIN C., "La RSE comme facteur de cohésion interne en situation de crise ", La Communication de Crise ESSensible 18, 2009.

GABRIEL P., « Le marketing comme moyen de légitimation des entreprises dans une perspective de développement durable ", Décisions Marketing 29, 2003.

FRAENKEL B.et LEGRIS-DESPORTES C., Entreprise et Sémiologie, Paris, Dunod 1999.

LAVILLE E., L'entreprise verte, Paris, Village Mondial, 2002.

LEGRIS-DESPORTES C., COUTON-WYPOREK P., CAPRON P., TSALA-EFFA D., Etudes "Sémios " Enquêtes en Entreprise, Collection Sciences Humaines, Les 2 Encres, 2008.

LEGRIS-DESPORTES C., «Blogs et transparence », Revue Sigila 26, MSH, 2010.

LIBAERT T., RSE, « crise et communication ", Magazine La Communication de Crise ESSensible 18, 2009.

PASTORE-REISS E., NAILLON H., Le marketing éthique, Village Mondial, 2002.

MERMET G., « Profitons de la crise pour refonder la société », Le Monde 14/10/2008.

PARGUEL B. et BENOÎT-MOREAU F., Communication sociétale et capital Marque, Actes de XXIII ${ }^{\text {ème }}$ Congrès de l'AFM, 2007. 
QUINTON P., «Ecriture, Spécificités morphologiques des écrits électroniques », Actes du colloque Ecriture en ligne: Pratiques et communautés, Sous la direction de Brigitte Chapelain, Université de Rennes 2. CERCOR/CERSIC, 2002.

REGUER D., COUTON-WYPOREK et LEGRIS-DESPORTES C., Blogs, Médias Sociaux et Politique, Collection Sciences Humaines, Les 2 Encres, 2009.

SASSI H., Stratégie de l'innovation et sémiotique du positionnement marketing, Thèse de l'université de Limoges, 2007.

SEMPRINI A., Le marketing de la Marque, Editions Liaisons, 1992.

SEMPRINI A., La marque, une puissance fragile, Vuibert, 2005.

SEMPRINI A., Analyser la communication 2, Collection Champs visuels, L'Harmattan, 2007.

Résumé : Cet article envisage les apports de la socio-sémiotique à l'analyse des pratiques communicationnelles et organisationnelles de la relation client, en abordant la question centrale de l'hétérogénéité médiatique et discursive ainsi que la nécessaire prise en compte des contextes institutionnels et socio-culturels. Il montre en quoi et comment les analyses des communications d'entreprises évoluent nécessairement, tant en termes de problématiques que sur le plan méthodologique.

Mots-clés : socio-sémiotique, sémiologie, entreprise, communication, relation client, interactions, internet, hétérogénéité discursive

Abstract : This article deals with how sociology and semiotic can help in the analysis of communication and management practices in client relationship. It speaks about the heterogeneity of discursive register and the heterogeneity of communication medium, as much as how important it is to focus on the type of institution, society and culture. It demonstrates how the analysis of the communication of companies changes from a problematic and methodological standpoint.

Keywords : socio-semiotics, semiology, firm, communication, customer relation, interactions, Internet, discoursive heterogeneity 